Velimir D. Mladenović

Univerzitet u Novom Sadu

Filozofski fakultet

Univerzitet u Poatjeu

Doktorska škola: Humanités

ITEM — Équipe Aragon https://doi.org/10.18485/analiff.2020.32.1.8

821.133.1.09 Арагон Л.

Originalni naučni rad

Primljen: 17.03.2020.

Prihvaćen: 11.06.2020.

\title{
LOUIS ARAGON ET LA RÉCEPTION DE SON OEUVRE DANS LE MILIEU YOUGOSLAVE ET SERBE DE 1945 À NOS JOURS
}

Cet article examine les relations personnelles, politiques et littéraires entre Louis Aragon, un des plus grands poètes français du $\mathrm{XX}^{\mathrm{e}}$ siècle, et les poètes et intellectuels yougoslaves et serbes, depuis la fin de la Deuxième Guerre mondiale jusqu'aujourd'hui. En prenant comme point de départ la présence de la Yougoslavie dans les œuvres d'Aragon publiées durant cette période, ainsi que ses relations personnelles avec les auteurs serbes, l'article montrera que la politique joue un rôle crucial dans le rapprochement entre Aragon et les intellectuels yougoslaves, en particulier avec Marko Ristić. L'article expliquera également l'éloignement d'Aragon d'avec la politique yougoslave et ses anciens amis après la Résolution du Kominform, puis mettra en évidence le reflet de ses pensées dans ses écrits et l'influence de la politique dans la réception de son œuvre dans le milieu yougoslave et serbe.

Mots clés : Louis Aragon, Marko Ristić, transfert culturel, réception, communisme

\section{LOUIS ARAGON ET LA POLITIQUE YOUGOSLAVE, DE 1945 À LA RÉSOLUTION DU KOMINFORM}

$$
\begin{array}{r}
\text { Il n'y a pas de lumière sans ombre. } \\
\text { Louis Aragon }
\end{array}
$$

Louis Aragon est présent dans la culture yougoslave et serbe depuis l'époque surréaliste, pendant laquelle les poètes et intellectuels serbes développent des relations personnelles et professionnelles avec le poète

*_velimirmladenovic@gmail.com / velimir.mladenovic@univ-poitiers.fr 
français. Entre 1924 et 1939 se produit un véritable transfert culturel et littéraire réciproque. Aragon connaît la poétique surréaliste de ses amis de Belgrade; il est également informé de leurs problèmes liés à la situation politique en Yougoslavie, sous l'emprise de la dictature du roi Alexandre. Il intègre dans ses écrits des informations sur la Yougoslavie et sur Belgrade auxquelles il a eu accès, tandis que son œuvre ne reste pas inaperçue auprès des auteurs serbes et dans les revues littéraires serbes d'avant-garde. Après la Deuxième Guerre mondiale, l'œuvre d'Aragon devient plus visible, et sa présence même sur les rivages du milieu yougoslave et serbe mérite d'être étudiée et présentée, à plusieurs niveaux. C'est pourquoi la première partie de notre article commence par présenter les relations franco-yougoslaves dans les années suivant la Deuxième Guerre mondiale, pendant lesquelles Aragon et les intellectuels yougoslaves et serbes se sont rapprochés, puis insiste sur les relations personnelles avec Marko Ristić, ainsi que sur la position d'Aragon face à la Yougoslavie et sa politique. La deuxième partie de notre article examine l'éloignement du poète français de ses amis yougoslaves, causé par la Résolution du Kominform, et la présence discrète de la Yougoslavie dans ses romans. Enfin, la troisième partie examine la réception de l'œuvre d'Aragon dans le milieu culturel yougoslave et serbe, de 1945 à nos jours.

La Deuxième Guerre mondiale éloigne le poète français des Balkans et de ses anciens amis surréalistes. Nous n'avons pas d'informations quant à d'éventuelles relations entretenues avec les intellectuels de cette région de l'Europe, pendant cette période particulièrement dure pour Aragon et sa femme Elsa Triolet. Le célèbre couple d'écrivains se concentre sur la Résistance intellectuelle en France, notamment par la création de journaux culturels clandestins ${ }^{1}$. Aragon publie des poèmes qui chantent la France, la gloire et la Résistance, tandis que Triolet écrit des nouvelles de guerre ${ }^{2}$. Après ces années noires, Aragon est considéré dans le milieu culturel français comme l'un des plus grands poètes en langue française de la Résistance. $^{3}$

$1 \quad$ Le couple crée le journal Les Étoiles et Aragon fonde Les Lettres françaises avec Jacques Decour.

2 Avec le recueil de nouvelles Le Premier accroc coûte deux cents francs, Elsa Triolet devient la première femme à obtenir le prix Goncourt en 1945.

$3 \quad$ Il ne faut pas oublier qu'Aragon est le premier poète de langue française qui introduit le mot «Auschwitz» en poésie. 
Dans les années d'après-guerre, les relations politiques et culturelles franco-yougoslaves évoluent dans un contexte sociopolitique, surtout marqué par l'orientation antifasciste des deux pays. Traducteur et promoteur d'Aragon en Serbie à l'époque surréaliste, Marko Ristić est nommé premier ambassadeur de la Nouvelle Yougoslavie en France. Dans la capitale française, l'ambassadeur entretient de bons rapports avec les intellectuels et les hommes politiques français, avec les anciens surréalistes et résistants, et surtout avec Louis Aragon et Elsa Triolet. Ceux-ci lui rendent régulièrement visite à la résidence de l'ambassade yougoslave à Paris, lors de cérémonies officielles. Plusieurs documents témoignent de ces rencontres amicales : en particulier, les ouvrages offerts par Louis Aragon et Elsa Triolet à Marko Ristić ${ }^{4}$, dédicacés et complétés par des notes manuscrites en bas de page. À plusieurs reprises, le journal Les Lettres françaises, fondé par Aragon, rend compte des activités de l'ambassade yougoslave : des articles informent des réceptions et des invitations des personnalités de gauche. Une autre preuve de cette amitié, et de l'activité de Marko Ristić en matière de diplomatie culturelle, est la publication en mai 1946 d'un article dans Les Lettres françaises, sous le titre «Liberté au peuple», dans lequel il décrit les années noires sous la botte allemande dans les Balkans. Cet article est le premier texte signé dans cet hebdomadaire par un auteur serbe et yougoslave; il est présenté dans ses pages comme un éclairage important sur un pays qui n'est pas suffisamment connu dans le miroir français :

«Nous sommes heureux de publier aujourd'hui l'article qu'il écrivit à Belgrade au lendemain de la Libération. Marko Ristich donne de son pays une image qui passionnera des amis français de Yougoslavie». (Ristić, 1946 : 1)

Les rencontres entre Aragon et Ristić suscitent également la publication d'un texte d'Aragon dans le numéro suivant des Lettres françaises, à propos de la culture et de la littérature ${ }^{5}$ de ce même pays :

4 Ces livres sont très précieux pour une nouvelle interprétation des idées d'Aragon, surtout par rapport au surréalisme. Voir Velimir Mladenović, Les Dédicaces d'Aragon au couple Ristić, Paris, Éd. Cahiers, 2019, 166-185.

5 L'article d'Aragon a été le premier sur la littérature serbe publié dans l'hebdomadaire Les lettres françaises. Voir plus sur ce sujet : Velimir Mladenović, La Littérature 
«À Paris comme à Rebesinje». Aragon y évoque, à l'attention des lecteurs français, ses amis surréalistes et l'histoire yougoslave. Cet article est un document rare, dans lequel il présente son expérience personnelle de la culture serbe et yougoslave : il y déclare que «entre nos deux pays cette communauté de rêves» a rendu «indestructible l'amitié».

«Plus tard dans ce monde fou d'après l'autre guerre, j'ai connu des Serbes, des garçons de mon âge, qui avaient les mêmes passions, les mêmes folies que moi. L'un d'eux est aujourd'hui à Paris, ambassadeur de son pays. Un autre est général et chef d'état-major de Tito là-bas. Oui, nous avons eu beaucoup de rêves en commun. Nous avons contemporains et camarades, cherché dans le même temps notre voie, par les mêmes chemins aberrants.» (Aragon, $1946: 5$ )

Cette politique culturelle d'association avec les intellectuels français se manifeste également sur le sol yougoslave, à travers les visites des écrivains étrangers et leur présence dans le milieu culturel et politique yougoslave. La Nouvelle Yougoslavie décide de s'orienter vers l'Occident; elle revendique également devant le monde son orientation antifasciste. Plusieurs écrivains français donnent des conférences à Belgrade : Paul Éluard en 1946, et Jean-Richard Bloch à l'automne de la même année. Ces écrivains diffusent dans le cadre européen des informations sur le pays qu'ils visitent et leurs impressions personnelles. En juin 1947, à l'invitation officielle de l'Associations des écrivains yougoslaves, Louis Aragon et Elsa Triolet visitent la Yougoslavie et y résident dix jours. Dans la capitale, le célèbre couple se promène avec les plus grands écrivains yougoslaves de l'époque : Ivo Andrić, Čedomir Minderović, Milan Bogdanović, Dušan Matić et Oskar Davičo. Ils visitent l'Académie de cinéma et échangent avec les étudiants. Ils se rendent dans les villes de Novi Sad et Sarajevo, sur la montagne d'Avala, dans la région de Šumadija, et visitent les travaux du chemin de fer Šamac-Sarajevo. À cette dernière occasion, le poète ne cache pas à la presse yougoslave sa forte impression, de se trouver au milieu des jeunes yougoslaves construisant un pays dévasté. ${ }^{6}$ Louis Aragon et Elsa Triolet assistent avec le président de la Tchécoslovaquie à une réception serbe dans Les Lettres françaises, Novi Sad, Matica srpska, 2019, pp. 55-68. 
chez le président de la République yougoslave. La liste des lieux visités et des personnalités avec qui ils se sont entretenus est impressionnante; elle nous invite à considérer cet événement, non pas comme une visite ordinaire d'écrivains étrangers, mais plutôt comme une réception officielle culturelle et politique, visant de démontrer aux invités et, à travers eux, au public français, l'orientation antifasciste de la Yougoslavie. Lors de ce voyage, au siège de l'Association des écrivains yougoslaves à Belgrade, Aragon tient une conférence de presse sur la littérature française. Sur la question des caractéristiques de la littérature française d'aujourd'hui, Louis Aragon déclare qu'il serait faux de considérer la littérature française de ce temps comme une chose entièrement neuve : «Elle est une suite de l'ancienne et grande littérature française et elle a eu de petites variations. » (Borba, 1947 : 3) Pendant cette conférence, il explique que deux phénomènes se sont produits en littérature entre les deux guerres : le surréalisme et la poésie pure, dont le représentant est Paul Valery; puis, après la Deuxième Guerre mondiale est apparu l'existentialisme, incarné en Jean-Paul Sartre. Il cite les écrivains devenus célèbres pendant la guerre : Vercors, Elsa Triolet, Claude Aveline et Claude Morgan.

En tant que connaisseur de la culture yougoslave et serbe, Aragon invite l'assistance à diriger l'attention du public français sur les traductions françaises du serbo-croate : il précise qu'il a déjà préparé pour la publication La Chronique de Travnik de Ivo Andrić et La Fausse de Ivan Goran Kovačić. ${ }^{7}$ Répondant à la question "Quelle est la position politique des écrivains français sur la crise de Trieste?», Aragon déclare que ceux-ci se tiennent aux côtés de leurs amis yougoslaves (Borba, 1947 : 3). Il faisait partie, en effet, des intellectuels français signataires du manifeste soulignant «l'urgence d'un règlement équitable de la question de Trieste», revendiquant une position favorable à la Yougoslavie (Les Lettres françaises, $1946: 2$ ).

«Il est certain qu'à Trieste même des Italiens ont, encore ainsi, reçu les armées de Tito. Ce parallèle à son prix. J'adjure ici mes amis italiens de le méditer. Je les adjure de ne pas servir

$7 \quad$ Ce poème a été publié en France en 1948. Marko Ristić l'a traduit en français. Picasso publie un burin et Paul Éluard, en guise de préface à ce même volume, écrit le poème «Tombeau de Goran Kovatchich». 
des intérêts obscurs et qui ne sont pas italiens en mêlant leurs voix aux voix de leurs meurtrières d'hier, aux voix fascistes qui, étrangement soutenues par des moyens non italiens, lorsqu'elles s'élèvent à nouveau contre la France (ou la Yougoslavie), ont pour but premier de faire le rappel de toutes les forces noires, de tous les ennemis de l'homme, de tous les fauteurs de guerre [...].» (Aragon, $1946: 5$ )

\section{La position d'Aragon face à la Résolution du Kominform de 1948}

La littérature est une affaire sérieuse pour un pays, elle est au bout du compte, son visage.

Louis Aragon

Les tensions politiques entre les dirigeants communistes de la Yougoslavie et les autres partis communistes européens dans les années d'après-guerre se reflètent sur le comportement des auteurs français envers ce pays, seul pays communiste à n'être pas entré dans le pacte de Varsovie en 1948. Au cours de cette période, Aragon s'éloigne de ses amis belgradois et de la culture yougoslave. Nous envisageons trois explications à cette brouille. En premier lieu, le poète ne pardonne pas aux écrivains yougoslaves les insultes adressées à Elsa Triolet dans le premier numéro de Književne novine de Belgrade, au moment où les communistes yougoslaves se démarquent de la politique de Staline. Dans cet article, l'auteur yougoslave Milan Bogdanović accuse le «regard superficiel» porté sur un pays idéologiquement proche, que Triolet aurait manifesté dans son reportage sur la Yougoslave. La deuxième possibilité, selon le premier biographe d'Aragon, Pierre Daix, sont les insultes des députés communistes yougoslaves adressées à la Résistance française lors de la première réunion du Kominform, en septembre 1947 (voir : Daix, 1975 : 360). Quant à la troisième explication, elle se réfère à la position politique d'Aragon, resté fidèle au parti communiste français au moment de la rupture entre Tito et Staline.

Nous affirmons avec certitude que la politique est indissociable du travail d'Aragon et, quand l'amitié avec les auteurs serbes se transforme en cauchemar, en 1948, Aragon écrit le deuxième tome de son roman 
Les Communistes (cinquième volume du cycle Le Monde réel), «où commencent d'apparaître les conséquences de la rupture entre Staline et Tito et du procès Rajk qui en découlent» (Daix, 1994 : 450). Il reste fidèle à son parti qui considère, pourtant, la Yougoslavie comme un pays de «traîtres ». Dans la presse française, il donne son opinion sur le procès de Rajk (secrétaire général adjoint du parti des travailleurs hongrois, exécuté vers la fin de l'été 1949) et sur les évènements survenus en Hongrie. Il condamne ouvertement le président de la Yougoslavie, en accusant la presse yougoslave de propagande :

«Nous avons des raisons de croire que les fauteurs de guerre comptaient sur un nouveau conflit en 1949. Au printemps de cette année, en effet, une révolte devait éclater en Hongrie, préparée par des espions américains et la clique de Tito. Mais les Hongrois saisirent à temps les conspirateurs à la gorge. » (L'Intransigeant, $1949: 5)$

Selon Dominique Desanti, Aragon n'est pas aussi sévère envers la politique yougoslave dans ses conversations privées. Au moment où Desanti publie une brochure anti-titiste ${ }^{8}$, Aragon déclare à son amie : «Écoute... Je n'ai pas besoin de te rappeler que sur Tito nous avions Elsa surtout — vu clair avant que l'Affaire éclate.» (Desanti, 1983 : 343)

«Il y avait eu un incident avec ce parti yougoslave qu'ils appelaient Ligue, je crois, hein? Écoute, les démocraties populaires, que savons-nous de leurs dirigeants? Ils ont, de toute façon, peu d'expérience du pouvoir. Et même chez nous. Écoute... à ta place, je citerais Maurice (Thorez), là tu es sûre. Benoît (Frachon)?... tu y tiens? Bon. Les autres? À quoi bon tous ces noms?» (Desanti, $1983: 344)$

À propos de cette publication de Desanti, Aragon donne son opinion : «Un livre, c'est fait pour durer en principe. Alors... si des remous... tu vois ce que je veux dire? Hein? Les noms...?» (Desanti, 1983 : 344) Une telle attitude politique se manifeste même dans ses romans. L'auteur considère désormais son ancien ami Jean Cassou (qui a pris position du côté de la politique yougoslave) comme un adversaire. En se rappelant ses 
amis dans La Mise à mort (1964), et plus particulièrement une rencontre avec Cassou, Tzara et Crevel en juin 1935, Aragon modifie ses souvenirs, ainsi que les sentiments et la responsabilité d'un personnage qu'il qualifie de «morcelé»: Jean Cassou". «La mémoire d'Aragon fonctionne comme les photos officielles du P.C., d'où sont gommés les visages des dirigeants en disgrâce» (Desanti, 1983 : 246). Plusieurs années après sa visite en Yougoslavie, Aragon déclare : «Je le pressentais, nous avons eu une très mauvaise impression, là-bas » (Desanti, 1983 : 330) et il démissionne de l'association culturelle France-Yougoslavie en même temps que d'autres écrivains français (tels Paul Éluard et Elsa Triolet).

Les références à la culture yougoslave dans son œuvre se font plus discrètes, mais ne disparaissent pas. La situation évolue après la réconciliation entre Khrouchtchev et Tito (1955) : même si Aragon n'est toujours pas favorable à la politique yougoslave, cet événement nouveau l'incite à faire parler ainsi son personnage d'étudiant yougoslave, dans son roman La Mise à mort :

«Pour lui, il n'aurait sans doute jamais imaginé tout seul que ce put être un Yougoslave. Mais pourquoi cela ne serait-il pas un Yougoslave? Un Yougoslave, cela se tue comme un autre.» (Aragon, $1965: 356$ ) [...] «Ce n'est pas là que le crime a été perpétré, puisque je n'y suis pas entré, moi. Ça ne fait rien, je regrette la Yougoslave. Parce que la victime de la rue FrançoisMiron, à part qu'il avait sur lui un pull-over acheté à Marseille, on ne sait rien de lui. Peut-être c'était un Yougoslave tout de même! (Aragon, $1965: 356$ )

Dans son roman Blanche ou l'oubli (1967), Aragon place l'un de ses personnages à Zagreb, c'est-à-dire dans une ville yougoslave : "[...] en raison d'un match à Zagreb en vue pour six ou sept semaines plus tard." (Aragon, 1967 : 13) Même s'il s'éloigne de ses anciens amis, surtout de Marko Ristić, les documents que nous avons pu consulter ${ }^{10}$ montrent qu'il

9 À partir de 1947, Cassou prend ses distances vis-à-vis du stalinisme, puis rompt définitivement en 1949, au retour d'un voyage en Yougoslavie. Il était président de l'association «France-Yougoslavie». Suite à son article «La Révolution et la vérité» publié dans la revue Esprit en 1949, il est attaqué par les communistes. André Wurmster publie un livre contre Cassou : Réponse à Jean Cassou, ENC, 1950.

10 Louis Aragon et Dušan Matić se sont rencontrés pour la dernière fois quelques 
reste en relation professionnelle avec le poète et intellectuel serbe Dušan Matić ${ }^{11}$ jusqu'en 1970. Ce dernier lui rend visite à Saint-Arnoult-en-Yvelines et un bloc thématique lui a été accordé dans Les Lettres françaises ${ }^{12}$ en 1968. Un entretien pour l'hebdomadaire yougoslave NIN datant de 1977 nous reste comme un témoignage de l'évolution de l'opinion politique d'Aragon envers la Yougoslavie et ses anciens camarades. Le poète y déclare qu'il ne comprenait pas le comportement des Russes vis-à-vis de la Yougoslavie. Il avoue également qu'il était impressionné par l'éloignement de la Yougoslavie de la politique de l'URSS. «Je sais ce que vous me demandez - répondit Aragon - j'ai visité votre pays en 1947, et votre séparation avec l'URSS s'est produite un an plus tard.» (Stanišić, 1977 : 33) Au sujet des dirigeants responsables de cet acte politique, il déclare qu'ils «étaient courageux, peut-être des héros.» (Stanišić, $1977: 33$ )

\title{
III. LA RÉCEPTION DES CEUVRES D'ARAGON EN YOUGOSLAVIE DE 1945 À NOS JOURS
}

\author{
La fiction ne suffit pas à caractériser le roman, \\ mais un certain rapport entre cette fiction et la réalité.
}

Louis Aragon

Après avoir vu la place importante occupée par la culture et la politique yougoslaves dans la vie professionnelle et dans plusieurs œuvres d'Aragon, nous nous penchons à présent sur la réception de l'œuvre de cet écrivain dans le milieu yougoslave et serbe, depuis la fin de la Deuxième Guerre mondiale jusqu'à nos jours. Cette réception est appréciée surtout à l'aune des traductions de ses œuvres, que nous pouvons diviser en trois grands groupes : les romans, la traduction de choix de poèmes, la traduction d'articles publiés dans des revues littéraires. Son roman Les Cloches de Bâle (1934) a été traduit en serbe relativement tard, c'est-à-dire en 1946 (publié par la maison d'édition Kultura à Zagreb). Une deuxième

mois avant la mort d'Elsa Triolet (1970). Les deux poètes étaient en correspondance. Voir plus sur ce sujet : Velimir Mladenović, Louis Aragon et Dušan Matić : une amitié inachevée (deux lettres inédites), Filološki pregled, n 46, v. 2 (2019), pp. 141-146.

11 Cet écrivain serbe salue l'œuvre de son ami français. Voir plus de détails : Dušan Matić, Jedan vid francuske književnosti, Beograd, Prosveta, 1952, pp. 195-199.

12 Voir plus sur Dušan Matić dans Les Lettres françaises : Velimir Mladenović, La Littérature serbe dans Les Lettres françaises, Novi Sad, Matica srpska, 2019, pp. 62-68. 
édition paraît en 1947 à Belgrade, tirée à cinq mille exemplaires. La même année est publiée la traduction serbe du recueil de nouvelles de guerre Servitude et grandeur des Français (Međunarodna knjižarnica, Belgrade). Malgré les polémiques entre Aragon et les poètes yougoslaves, le nombre de ses ouvrages traduits ne diminue pas. Parmi ses principaux romans : Les Beaux quartiers est publié à Zagreb (Nakladni zavod Hrvatske), tandis que La Semaine sainte en 1961 (à Subotica, à Belgrade, dans la maison d'édition Minerva). Aurélien a été traduit et publié en Yougoslavie en 1963 (Novi Sad, Matica srpska). Le roman surréaliste Le Paysan de Paris (1926), quant à lui, entre très tard dans la culture yougoslave et serbe, en 1964 seulement (Belgrade, Prosveta).

Les poèmes d'Aragon dans les années d'après-guerre ont été publiés dans des revues littéraires, ainsi qu'en recueils présentant des sélections. Le traducteur Nikola Trajković publie le recueil Izmišljene ruže [Les roses inventées] en 1962, tandis que Milovan Danojlić traduit Velika radost [La Grande Gaité] en 1974 avec une préface sur la poésie d'Aragon. Nikola Trajković publie un autre recueil : Poveriću ti veliku tajnu [Je vais te dire un grand secret] en 1982 et 1986, au tirage de cinq mille exemplaires. Ces deux éditions sont illustrées de photographies du poète à différentes époques. Par ailleurs, des poèmes d'Aragon sont proposés dans les anthologies suivantes: Iskra, ljubav, reč [Étincelle, amour, parole] (éditeur $\mathrm{S}$. Soldatović). Deux poèmes en traduction serbe, «Les lilas et les roses» et «Richard II Quarante», figurent dans Antologija novije francuske poezije [Anthologie de la nouvelle lyrique française] composée par le poète Ivan V. Lalić en 1966. En 1960, Antologija savremene francuske poezije [Anthologie de la poésie française contemporaine] présente «Un air d'octobre» et «Richard II Quarante». Enfin, sept poèmes d'Aragon, dans la traduction de Mirjana Vukmirović, sont présentés dans Antologija francuske poezije XX veka [Anthologie de la poésie française du $X X e$ siècle] : "Poème à crier dans les ruines», «Parti-pars», "La Force», «Vie de Jean-Baptiste A», «Air du temps», «Les approches de l'amour et du baiser», «Elsa au miroir». Les choix de poèmes rassemblés dans ces anthologies font preuve d'un manque de diversité : presque tous les poèmes d'Aragon traduits en serbe sont des poèmes d'amour, et ce sont ceux pour lesquels Aragon est connu à travers le monde. Ces sélections ne rendent pas hommage à son autre facette : le poète communiste et résistant. 
Malgré toutes ces traductions, la réception de son œuvre en Yougoslavie reste presque inaperçue. Elle n'est présentée que dans des revues littéraires, surtout dans le Journal littéraire [Književne novine] de Belgrade. On y trouve six textes et traductions dédiés à Aragon entre 1948 et 1972. Le premier article qui lui est consacré, écrit par Čedo Kisić, montre que la politique sépare les surréalistes français des surréalistes serbes. À travers ce texte, l'auteur serbe s'adresse au poète français : il lui explique que le parti ne lui a pas rendu les «couleurs de la France»; ou bien, si c'était le cas, alors les couleurs de ce pays ont vraiment perdu la valeur qu'elles possédaient depuis des siècles. (Kisić, 1951 : 2) Dans un autre texte dédié à l'écrivain romantique Victor Hugo, le même auteur mentionne une conférence donnée par Aragon à la Sorbonne en 1956, puis fait un état des lieux des livres imprimés en URSS : il déclare que le nombre des livres d'Hugo dépasse tous les autres, avec un nombre de 96300000 exemplaires. (Jeremić, 1959 : 5) La même année, quand Aragon publie en France l'anthologie de la poésie d'Hugo ${ }^{13}$, des séances en l'honneur de l'écrivain des Misérables sont organisées en Chine, en présence de Mao Tsé-Toung. Ces événements ont été salués dans les revues littéraires. Aragon a été considéré par Bertolino comme le poète contemporain qui a su redécouvrir le poète de l'époque romantique. ${ }^{14}$

Un article de Dragan M. Jeremić intitulé «Književna panorama Pariza» [Le Panorama littéraire parisien] de 1959 présente Aragon comme un écrivain reconnu dans son pays, et considère que son dernier roman, La Semaine Sainte, est une œuvre très importante, quand bien même on pourrait ne pas l'apprécier personnellement, qu'elle mérite d'être couronné par le prix Goncourt. Mais, cette année-là, le jury a préféré le décerner à un auteur moins célébré.

Le poème «Noć mladih ljudi»a été traduit et publié dans Književne novine par Nikola Trajković, qui annonce en même temps la parution d'un recueil de poèmes d'Aragon en serbo-croate. (1960: 5) Deux autres traductions sont présentées dans les pages de ce journal culturel : celle de «Magnitogorsk»en 1967, par Trajković, et «Les Chambres» par Lela Matić en 1971.

13 L'auteur se réfère au livre : Avez-vous lu Victor Hugo? Anthologie poétique commentée par Aragon, Les éditeurs français réunis, Paris 1952.

14 Voir plus sur ce sujet : Književnost, 1964 : 317-334. 
Le roman Blanche ou l'oubli est accueilli avec enthousiasme dans les pages de ce journal. L'article critique de Matvejević clame que ce roman est aussi attendu «que le roman de Malraux Anti-mémoires » (Matvejević : 1968 : 4) et se demande comment Aragon, le plus éminent promoteur du réalisme social en France, s'est orienté, à l'âge de soixante-dix ans, vers un roman d'avant-garde. Il compare Blanche ou l'oubli au roman Les Gommes d'Alain Robbe-Grillet. Il s'attache également au prénom du personnage central, Blanche, qui provient du roman de son épouse Elsa Triolet LunaPark. Il souligne par ailleurs les traits communs entre le narrateur du récit, qui porte le nom de Gaiffier, et l'auteur lui-même, ainsi que leur date de naissance identique.

Après la résolution du Kominform, le premier entretien avec Aragon est publié dans Književne novine, accordé à Julija Najman en 1968, a eu lieu au domicile du poète à Saint-Arnoult-en-Yvelines : le «moulin». La journaliste décrit le parc, l'entourage du couple d'écrivains, mais également le comportement de celui-ci. Elle est venue au moulin avec un exemplaire du Paysan de Paris. Dans cet entretien, Aragon avoue qu'il s'est toujours considéré comme réaliste, que le surréalisme est toujours un réalisme, et que l'écriture automatique représentait un voyage en soi-même. En conclusion de leur conversation, le poète interroge la journaliste yougoslave au sujet de trois surréalistes belgradois : Matić, Ristić et Koča Popović. Il se rappelle les jours où Popović est venu à Paris à l'âge de vingt-cinq ans, lorsque le jeune surréaliste serbe lui avait avoué qu'il n'était venu à Paris que pour lui. Puis, Aragon déclare qu'il est en train de travailler sur un roman sur Matisse et qu'il ne perçoit pas la différence entre la prose et la poésie. Ce roman est présenté en quelques lignes, dans ce journal, comme un collage de textes critiques d'Aragon écrits entre 1941, l'année où il rencontre le peintre à Nice, et 1968. (Đorđević, 1972 : 11). Nada Đorđević indique que la plupart de ces textes ont déjà été publiés en France, mais qu'Aragon ajoute, dans ces deux gros volumes, ses commentaires nouveaux qu'il nomme des parenthèses. Ces écrits inédits occupent treize chapitres d'un ouvrage très illustré. L'auteur serbe souligne les différences de caractère entre Aragon et Matisse, en expliquant que le peintre se tenait en retrait lors de la Guerre, dans son atelier, tandis qu'Aragon a choisi de critiquer le monde, déterminé à prendre part à la résolution du conflit. 


\section{CONCLUSION}

Le monde est rempli de faux témoins. Louis Aragon

Le transfert culturel entre Aragon et les auteurs yougoslaves et serbes, évident et mutuel depuis presque un siècle, est une question complexe, car elle fait intervenir de multiples enjeux. Nous avons vu que ces relations se sont tissées à la fois sur le plan littéraire (en particulier, grâce aux affinités surréalistes d'avant-guerre) et dans les amitiés développées au fil des années avec plusieurs personnalités clés, puis sur la question politique, devenue centrale après-guerre ; surtout à cause de la resolution de Kominform. Nous avons constaté que malgré les polemiques politiques, nous trouvons des traces sur la culture yougoslave dans les écrits d'Aragon.

Quant à la réception, l'œuvre d'Aragon connaît des hauts et des bas en Yougoslavie et en Serbie, jusqu'aujourd'hui, mais les raisons de ces variations sont essentiellement extra-littéraires. La grande valeur artistique des romans et poèmes de cet écrivain, ainsi que la présence de la politique et de la culture yougoslaves et serbes dans ses textes, méritent une lecture et une étude plus objectives - centrées sur son œuvre. ${ }^{16} \mathrm{C}$ 'est pourquoi, au lieu d'une conclusion définitive, nous citerons l'article sur la mort d'Aragon publié dans le quotidien serbe Politika qui nous reste comme l'image sur Aragon, qui est à la fois un militant communiste avec ses illusions politiques et grand écrivain: «L'on sent cette influence dans la grande fresque politico-littéraire d'Aragon intitulée Les Communistes de 1949 à 1951 et dans la participation diffamatoire de ce grand écrivain, décédé aujourd'hui, dans la campagne de Staline contre la Yougoslavie et son indépendance, qu'Aragon a plus tard regrettées.» (Prlja, 1982:3) « Les pages du nouveau « Le Roman inachevé » et le poème le plus récent d'Aragon se trouvent à une frontière impressionnante de la découverte de l'essentiel de l'inconscient, du caché et du mystérieux en tant que source de cette vraie inspiration poétique irrationnelle. $\gg{ }^{17}(\operatorname{Prlja}, 1982: 3)$

15 L'auteur de cet article a organisé et animé un séminaire à l'École Normale Supérieure à Paris, en 2018, sur la réception de Louis Aragon et Elsa Triolet en Serbie. Voir plus sur ce sujet : https://www.fabula.org/actualites/la-reception-de-louis-aragon-etelsa-triolet-en-serbie_83080.php

16 L'auteur de cet article a traduit cet article en français. 


\section{REFERENCES}

\section{Sources}

1. Aragon, L. (12 juillet 1946). Amica Italia. Les lettres françaises, p. 5.

2. Aragon, L. (1965). La Mise à mort. Paris : Gallimard.

3. Aragon, L. (1967). Blanche ou l'oubli. Paris : Gallimard.

4. Ristić, M. (10 mai 1946). Liberté au peuple. Les Lettres françaises, p. 3.

5. Aragon, L (14 mai 1946). À Paris comme à Rebesinje. Les Lettres françaises, p. 5.

\section{Références bibliographiques}

1. (14 juin 1947). Luj Aragon i Elza Triole o današnoj francuskoj književnosti. Borba, p. 3.

2. (21 okt.1960). Noć mladih ljudi. Književne novine, p. 5.

3. (28 okt. 1967). Magnitogorsk. Književne novine, p. 10.

4. (8 mai 1971). Sobe, Književne novine, p. 7.

5. Daix, P. (1975). Aragon, une vie à changer. Paris : Seuil.

6. Daix, P. (1994). Aragon. Paris : Flammarion.

7. Desanti, D. (1983). Les Clés d'Elsa. Paris : E. Ramsay.

8. Đorđević, N. (1. nov. 1972). Aragonov roman o Matisu. Književne novine, p. 11.

9. Jeremić, M.D. (16 jan.1959). Književna panorama Pariza. Književne novine, p. 5.

10. Kisić, Č. (25 sept. 1951). Sjaj i beda Aragonov pokliča. Književne novine, p. 2

11. Les Lettres françaises (28 juin 1946), p. 2.

12. M.R (1947). Strani književnici na omladinskoj pruzi Šamac-Sarajevo. Književnost, nov.-déc. 1947, br. 11-12, p. 462.

13. Matić, D. (1952). Jedan vid francuske književnosti. Beograd : Prosveta.

14. Matvejević, P. (16 mars 1968). Louis Aragon, Blanche ou l'oubli, Književne novine, p. 4.

15. Mladenović, V. (2019). Les Dédicaces d'Aragon au couple Ristić. Cahier Aragon 2. Paris : Éd. Cahier.

16. Mladenović, V. (2019). La Littérature serbe dans Les Lettres françaises 1945 1970. Les relations littéraires et culturelles franco-serbes dans le contexte européen. Novi Sad : Matica srpska.

17. Mladenović V. (2019). Louis Aragon et Dušan Matić : une amitié inachevée (deux lettres inédites), Filološki pregled, ${ }^{\circ} 2$, pp. 141-146.

18. Prlja, A. (25 décembre 1982). Umro Luj Aragon, Politika, p. 3.

19. Stanišić, S. (1977). Ničeg se ne stidim. Nin, p. 33.

20. Književnost, (avril 1964) pp. 317-334. 


\title{
Velimir D. Mladenović
}

\section{LUJ ARAGON I RECEPCIJA NJEGOVIH DELA NA JUGOSLOVENSKOM I SRPSKOM KULTURNOM PROSTORU OD 1945. DO DANAS}

\begin{abstract}
Sažetak
Luj Aragon (1987-1982), jedan od najvećih francuskih pesnika XX veka, pristalica dadaističkog i jedan od osnivača nadrealističkog pokreta, od svog detinjstva bio je okružen Srbima, koji su se posle albanske Golgote školovanje i život nastavili u Francuskoj. U periodu između dva svetska rata ovaj autor ostvaruje bliski kontakt sa srpskim nadrealistima, upoznaje se sa jugoslovenskom i srpskom kulturom i politikom.

Međutim u periodu posle Drugog svetskog rata, ovom komustičkom piscu, nova Jugoslavija bila je ideološki bliska i Aragon obnavlja kontakte sa srpskim autorima, naročito sa Markom Ristićem. Zato ćemo mi u radu kroz novinske i propagandne članke koje je potpisao ovaj autor predstaviti njegov odnos prema jugoslovenskoj politici i kulturi i kako su lični kontakti sa srpkim autorima pomogli ovom piscu da bolje razume jugoslovenski kulturni prostor. Istražićemo i pokazati koji su razlozi naveli Luja Aragona da se udalji od srpskih pisca i intelektualaca posle rezolucije Imfombiroa 1948.

Budući da je kulturni transfer tekao u oba smera, pokazaćemo i da recepcija dela ovog autora na jugoslovenskom kulturnom prostoru u periodu od završetka Drugog svetskog rata do danas neposredno zavisi od politike.
\end{abstract}

Ključne reči : Luj Aragon, kulturni transfer, recepcija 